\title{
Protection anticorrosion par système duplex
}

\author{
Hervé Barréda ${ }^{(a),}$ Rolland Gallina ${ }^{(b)}$ \\ (a)Ministère de l'Equipement, des Transports, du Logement, du Tourisme et de la Mer- \\ CETMEF Agence d'Aix en Provence- \\ Chargé de la Corrosion et des Expertises Subaquatiques - Scaphandrier-plongeur professionnel. \\ (b)Conseil Général des Pyrénées Orientales, \\ Responsable Technique de l'Antenne Portuaire au port de commerce de Port-Vendres.
}

\section{Résumé}

L’enjeu économique important que représente la conservation du patrimoine à la mer, nous conduit à exposer une politique de protection anticorrosion par système duplex applicable sur les structures métalliques portuaires. Il s'agit de travaux relatifs à la construction du quai de l'Anse Gerbal à Port-Vendres (Pyrénées Orientales). La particularité de la méthode préventive réside dans le système choisi pour conserver les pieux métalliques. Cette technique novatrice de chemisage est destinée à protéger la zone d'éclaboussures des têtes de pieux contre la corrosion, les parties immergées étant protégées par anodes sacrificielles : c'est le système duplex : chemisage/protection cathodique. Il s'agit d'un exemple innovant en terme de prolongation de la durée de vie d'un ouvrage et en terme de diminution de frais et d'interventions ultérieurs.

\section{Abstract}

Important economic stake that the conservation of patrimony to the sea represents, leads us to expose a policy of corrosion protection for method of use duplex system on the harbour metal structures. It's work relating to the construction of l'Anse Gerbal quay in Port-Vendres (Pyrénées Orientales). The characteristic of this preventive method lies in the system chosen to preserve the metal piles. This innovative engineering of lining is intended to protect the splash zone from the heads of piles against corrosion, the immersed parts being protected by sacrificial anodes: a duplex system: cathodic. chemical/protection. It is an innovation in term of prolongation of the lifetime of the structure and in reduction for later expenses and further interventions

$\underline{\text { Mot-clés }}$ :Système duplex, anticorrosion, protection cathodique, chemisage, IVOR, ouvrage portuaire..

\section{Introduction}

L'objectif de l'article vise à considérer un système de protection anticorrosion et de mettre l'accent sur la nécessité de prévenir les phénomènes de corrosion dans le cadre de la surveillance et de la maintenance des ouvrages métalliques fixes en eau de mer[1]. La particularité du système réside dans la technique choisie pour protéger la zone d'éclaboussures et immergée des pieux métalliques porteurs. 


\section{Protection anticorrosion duplex : par chemisage et protection cathodique.}

\subsection{Présentation du chantier de Port-Vendres.}

\subsubsection{Principe du système de protection anticorrosion}

Toute structure métallique en contact permanent ou intermittent avec l'eau de mer va subir, à un certain rythme, des dégradations dès sa mise en place et tout au long de sa durée de vie. Il est donc préférable de prévenir ces phénomènes de corrosion dès la construction par un système de protection adapté qui va permettre de réduire le rythme de dégradation de l'ouvrage et ainsi éviter, pratiquement, toute réparation majeure et surtout toute mise hors service prématurée. La présente procédure de protection anticorrosion, s’inscrit dans le cadre d'une politique de prévention et concerne les pieux-tubes supports de l'appontement du quai de l’Anse Gerbal au port de Port-Vendres (Pyrénées Orientales).

Les parties immergées des pieux sont protégées par des anodes sacrificielles. Les parties hors d'eau et la zone d'éclaboussures sont protégées par mise en place d'un chemisage constitué d'un tube polyéthylène -PE- destiné à éviter tout contact entre les têtes de pieux et l'électrolyte.

Limite de la méthode : cette technique est envisageable lors de la construction d'un ouvrage, avant la construction du tablier et pour des mers à faible marnage.

\subsubsection{Origine de la protection : désordres sur le poste d'avitaillement}

Des mesures d'épaisseurs résiduelles par ultrasons réalisées sur les pieux-tubes métalliques du poste d'Avitaillement ont révélé une perte de métal de plus de $\sim 0,50 \mathrm{~mm} / \mathrm{an}$, ce qui est $\sim 5$ fois plus élevé que la moyenne mesurée en Méditerranée. A noter que les mesures ont été relevées sous le niveau des plus basses eaux, au milieu et en partie basses de tous les pieux. La constatation de ce phénomène exacerbé de corrosion a conduit le maître d'ouvrage à prendre des dispositions supplémentaires pour la construction du quai de l'Anse Gerbal. L'objectif est de protéger les pieux contre une diminution certaine de leurs sections qui présenterait, à court terme, des faiblesses préoccupantes pour la stabilité d'ensemble de l'ouvrage.

Causes probables ayant conduit à ces pertes de métal :

- présence, dans le fond, de câbles en acier inoxydable (câbles de chaluts) et de débris métalliques (tubes, cornières, plaques, ...) en contact avec les pieux, créant ainsi des couples galvaniques ;

- existence de courants vagabonds par du soudage effectué sur le chantier de carénage : le courant électrique a toujours tendance à circuler dans les conducteurs qui offrent une résistance minimale. Ceci se traduit par la circulation de courants non contrôlés qui protègent la structure quand ils vont de l'anode vers la cathode (on parle alors de polarisation cathodique), mais qui la corrode dans le cas inverse.

- écoulement d'eaux de lavage (certainement chargées en résidus «antifouling») provenant du chantier de carénage proche du poste d'avitaillement ainsi que des écoulements d'exutoires d'eau de pluie ou d'effluents urbains, ayant une influence sur la résistivité de l'eau de mer, qui est fonction de la température et de la salinité de l'eau. 


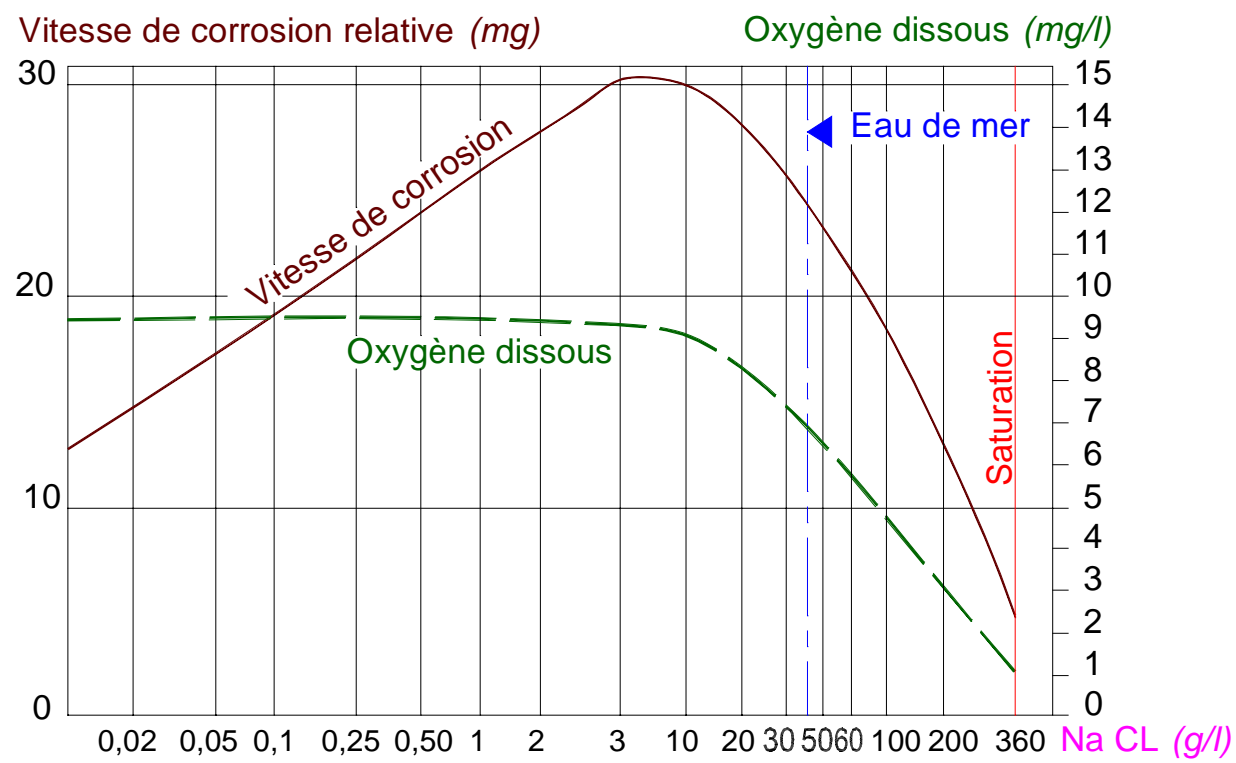

Figure 1 - Variation de la corrosion avec la salinité

On constate, selon André Hache[2] (figure 1), que l'attaque maximale se produit pour une teneur en chlorure de sodium -NaCl- d'environ 5 à $10 \mathrm{~g} / \mathrm{l}$, ce qui représente une teneur inférieure à celle habituelle en eau de mer, soit 27 g/l.

C'est pourquoi l'eau saumâtre (mélange eau salée/eau douce) est plus agressive que l'eau de mer seule[3]. Par ailleurs, les différences de concentration en sels dissous entre l'eau douce et l'eau salée seront favorables à la création de piles de concentration : l'attaque de l'acier sera alors plus rapide au contact de l'eau salée.

\subsubsection{Description de l'ouvrage}

Il s'agit d'un quai sur pieux dont la fonction est d'accueillir des navires de pêches de $30 \mathrm{x}$ $7 \mathrm{~m}$; le tirant d'eau est de 4,50 m. L’ouvrage (figure 3) est fondé sur deux files de pieuxtubes métalliques ( $\phi 863 \mathrm{~mm}$ de $15 \mathrm{~mm}$ d'épaisseur) supportant une dalle de béton armé de 0,30 m d'épaisseur. Les 8 pieux de la file arrière du quai sont dotés de tirants d'ancrage de $\phi$ $40 \mathrm{~mm}$ protégés par fourreau métallique. La file bord du quai est constituée de 9 pieux (la hauteur de la zone d'éclaboussure est de $0,65 \mathrm{~m}$ est la hauteur immergée des pieux avant est de 4,50 m). Au total, 17 pieux soutiennent la plate-forme.

\subsection{Protection anticorrosion des têtes de pieux par chemisage}

La disposition constructive consiste à préserver de la corrosion les pieux, par un système de protection mixte : chemisage en PE des têtes (figure 2) et anodes sacrificielles. La solution conservatrice retenue a pour objet de stopper les attaques dues au milieu agressif en abaissant le potentiel des pieux et en les isolants de l'électrolyte (eau de mer) sur leurs parties hautes. 

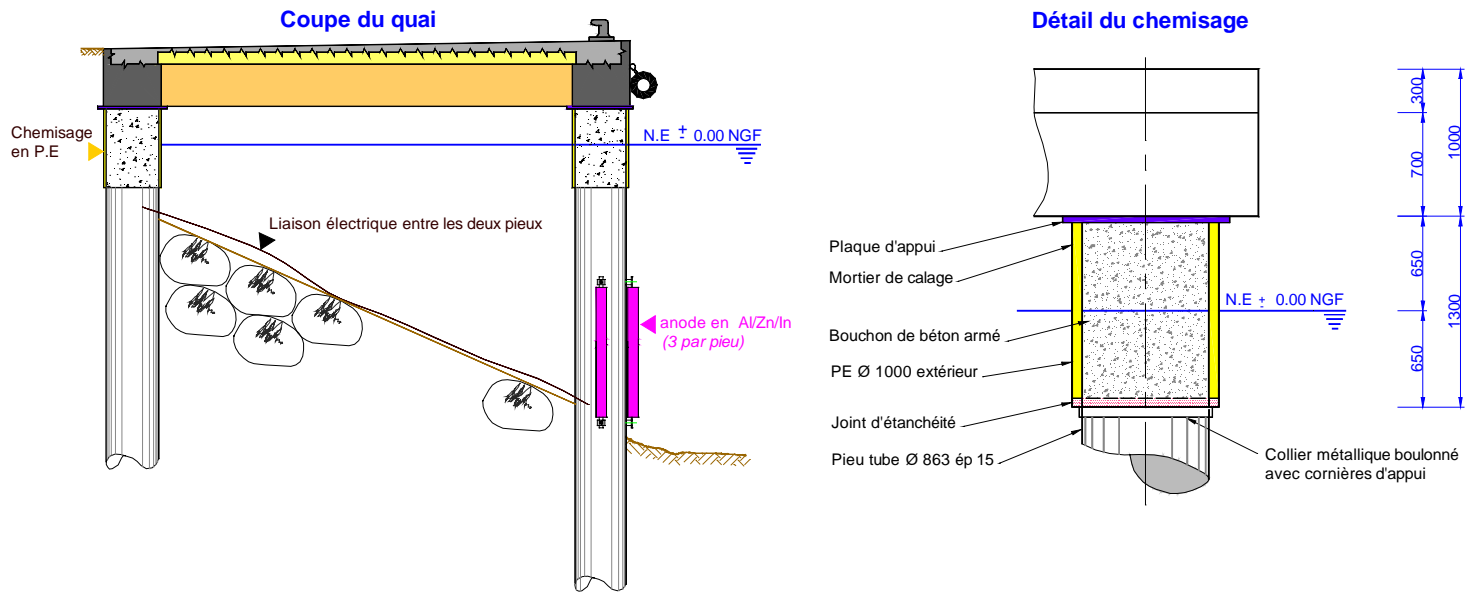

Figure. 2 - Principe du chemisage des têtes de pieux

Il est important de noter que cette technique ne peut être appliquée que lors de la construction du quai. Elle est donc mise en place après les opérations de battage des pieux et avant la construction de la dalle en béton armé. La protection anticorrosion, pour la partie située en zone d'éclaboussures, est assurée par des tubes PE constituants le chemisage extérieur des pieux.

L'espace annulaire existant entre le pieu métallique et le tube PE est rempli d'un mortier de calage à retrait compensé. L'étanchéité à la base du chemisage est établie par un joint gonflé (chambre à air de VTT -Vélo Tout Terrain-) mis en place par des scaphandriers. Le tube PE protège le pieu sur une hauteur de 1,30 $\mathrm{m}$ dont $0,65 \mathrm{~m}$ hors d'eau et $0,65 \mathrm{~m}$ sous l'eau (figure 2).

\subsubsection{Matériaux et moyens utilisés}

Le chemisage en PE de $\phi 1000$ mm extérieur pour une longueur de 1,30 m, le mortier de calage utilisé est du type Sikagrout 217 de SIKA. Le coulis est préparé dans l'unité malaxeur du type Cocentall et pompe d'injection.

\subsubsection{Méthodologie}

Avant toute mise en place sur les pieux battus, il est nécessaire d'effectuer des opérations à blanc hors d'eau similaires à celles décrites dans la méthodologie, afin d'éviter tous problèmes d'ordre technique pouvant survenir lors de la pose du chemisage in situ. Ainsi, il est possible de s'assurer de la faisabilité de la technique. Un collier métallique constitué d'un fer plat boulonné est descendu le long du pieu-tube jusqu'à la cote -0,70 NGF (Nivellement Général de la France). A noter que le collier est muni de cornières d'appui soudées. Positionné par les scaphandriers, le collier est bloqué à la cote susdite.

Un joint de caoutchouc gonflable est alors glissé le long du tube jusqu'au contact du collier métallique. Le tube de polyéthylène, servant de coffrage perdu, est élingué puis mis en place à l'aide d'une grue. Il est ainsi descendu jusqu'à appuyer sur les cornières métalliques d'arrêt. Rappelons que le collier est équipé de cornières d'appui pour : d'une part, centrer et d'autre part, pour soutenir le chemisage. Le joint est alors gonflé par les plongeurs réalisant ainsi l'étanchéité de la base du chemisage. A l'avancement, six pieux sont équipés avant de commencer l'injection du mortier de calage.

Le coulis d'injection est préparé à terre puis injecté depuis la surface avec un tube plongeur de $\phi 12 \mathrm{~mm}$. Il est injecté dans l'espace annulaire, entre chemisage et pieu, depuis le bas vers la haut. L'injection est menée alternativement d'un pieu à l'autre dans le but d’assurer un début de prise en fond de coffrage pour l'étanchéité du système. 
Elle se fait sous contrôle des plongeurs qui s’assurent de l'étanchéité, puis est poursuivie jusqu'au débordement du coulis en partie supérieure. Il est ensuite nécessaire de surfacer la partie supérieure du chemisage, afin que la plaque métallique chevêtre, puisse venir reposer sur une surface plane.

\subsubsection{Efficacité du système}

L'épaisseur d'enrobage est de 5,5 cm (espace annulaire) ce qui, associé à l'épaisseur de 1 $\mathrm{cm}$ du PE, confère au système une bonne résistance mécanique et aussi vis à vis des agents agressifs.

D’un point de vue électrochimique, l'acier est passivé. En effet, le $\mathrm{pH}$ du mortier est voisin de 13 ce qui met le pieu métallique à l'abri de la corrosion.

Enfin, la circulation de l'eau à l'interface métal/mortier est improbable car le système est adhérent et étanche.

\subsection{Protection cathodique des pieux par anodes sacrificielles $[4,5]$}

Tableau 1 - Protocole du dimensionnement de la protection cathodique.

\begin{tabular}{|c|c|c|c|c|}
\hline Surface d'un piet & $\begin{array}{c}\text { Densité du courant } \\
\text { de protection } \\
\left(\mathrm{m}^{2}\right)\end{array}$ & $\begin{array}{c}\text { Résistivité } \\
\text { du milieu } \\
\left(\mathrm{mA} / \mathrm{m}^{2}\right)\end{array}$ & $\begin{array}{c}\text { Potentiel théorique } \\
\text { de la structure Es } \\
(\Omega . \mathrm{cm})\end{array}$ & $\begin{array}{c}\text { Objectif de la } \\
\text { durée de vie de } \\
\text { la protection } \\
(\mathrm{V} / \mathrm{Ag} / \mathrm{AgCl})\end{array}$ \\
\hline \hline Immeres $)$
\end{tabular}

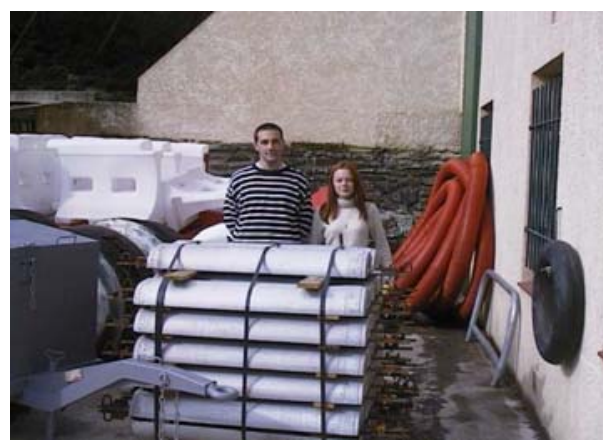

Figure 3 - Recette des anodes à la livraison

Récapitulatif

\begin{tabular}{|c|c|}
\hline Alliage anodique : & $\mathrm{Al} / \mathrm{Zn} / \mathrm{In}$ \\
\hline Nombre d'anodes : & 26 \\
\hline Nombre d'anode par pieux avant : & 3 \\
\hline $\begin{array}{l}\text { Forme de l'anode : } \\
\text { cylindrique }\end{array}$ & Semi- \\
\hline Diamètre de l'anode : & $\phi 250 \mathrm{~mm}$ \\
\hline Longueur de l'anode “La” : & $1120 \mathrm{~mm}$ \\
\hline Masse nette de l'anode : & $74 \mathrm{~kg}$ \\
\hline Masse totale anodique : & $1924 \mathrm{~kg}$ \\
\hline
\end{tabular}

Alliage anodique :

$\phi 250 \mathrm{~mm}$

$1120 \mathrm{~mm}$

$74 \mathrm{~kg}$

$1924 \mathrm{~kg}$

$\mathrm{kg} / \mathrm{A}$.an

\subsubsection{Vérification du fonctionnement de la protection cathodique}

La vérification réalisée (janvier 2002) sur les pieux après $\sim 3$ mois de fonctionnement révèle une moyenne de potentiel -E- de $-0,903 \mathrm{~V} / \mathrm{Ag} / \mathrm{AgCl} / \mathrm{eau}$ de $\mathrm{mer}^{(1)}$. Le dispositif mis en place préserve les pieux des effets de la corrosion, car il satisfait au niveau de potentiel minimal requis dans ce domaine -0,800 V/Ag/AgCl/eau de mer - selon la Norme NF EN 12473 du 062000 sur les principes généraux de la protection cathodique en eau de mer -.

\subsection{Coût des travaux de protection anticorrosion duplex}

Le prix total de l'ouvrage est de $1067143 €$. Le coût de la protection représente $~ 5,6 \%$ du prix de l'ouvrage, soit $\sim 60407 €$. La part du chemisage est de $40589 €$, soit $\sim 3,8 \%$ et la part de la protection cathodique est de $19818 €$, soit 1,8\% du montant total de l'ouvrage. 


\section{Conclusions \& Perspectives}

Cette étude nous amène à constater que la prévention anticorrosion ne fait pas l'unanimité. Le concepteur a une approche surépaisseur sacrificielle, alors que le «corrosionniste» a une approche protection cathodique ou système duplex (le coût est, respectivement, de $\sim 3$ à $~ 6 \%$ du prix total de l'ouvrage). La conséquence est que bon nombre de structures à la mer ne sont pas protégées de la corrosion. La combinaison des deux approches, dés la conception devrait être envisagée.

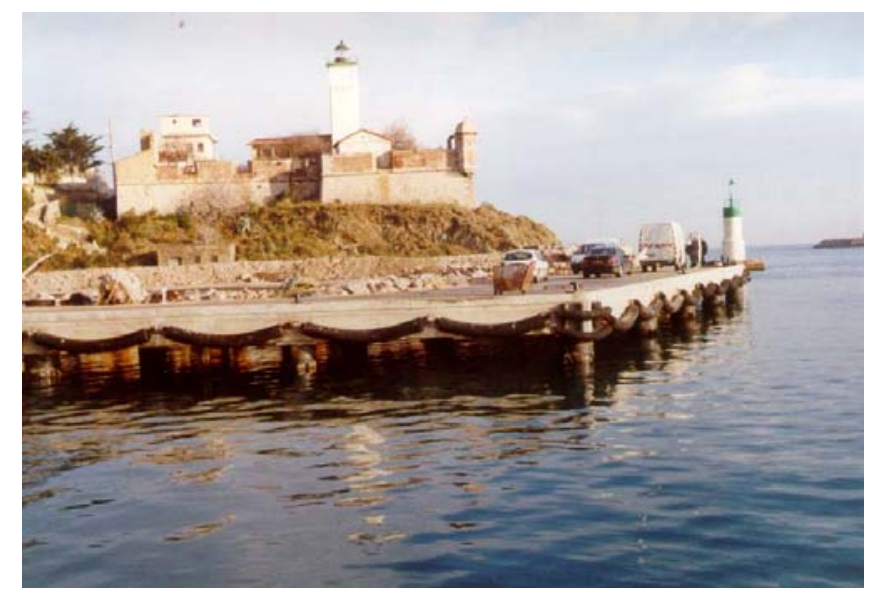

Figure. 4 - Vue d'ensemble du quai

Rappelons que la solution «maintenance au coup par coup», des structures fixes en eau de mer, s'avère souvent plus onéreuse et plus risquée en terme de stabilité de l'ouvrage. S'il est facile de caréner un navire en cale sèche, il faut trouver une solution 'sous l'eau' pour les structures portuaires fixes.

Aujourd'hui une étude lancée par le CETMEF sur la qualification de la méthode d'évaluation de l'efficacité anticorrosive de revêtements époxy applicables en immersion est en cours de validation pour une publication dans la Revue Technique du CETMEF.

Quoiqu'il en soit, n’est-il pas préférable de prévenir les risques de corrosion, et ce, dès la conception par différentes dispositions soit constructives soit anticorrosives?

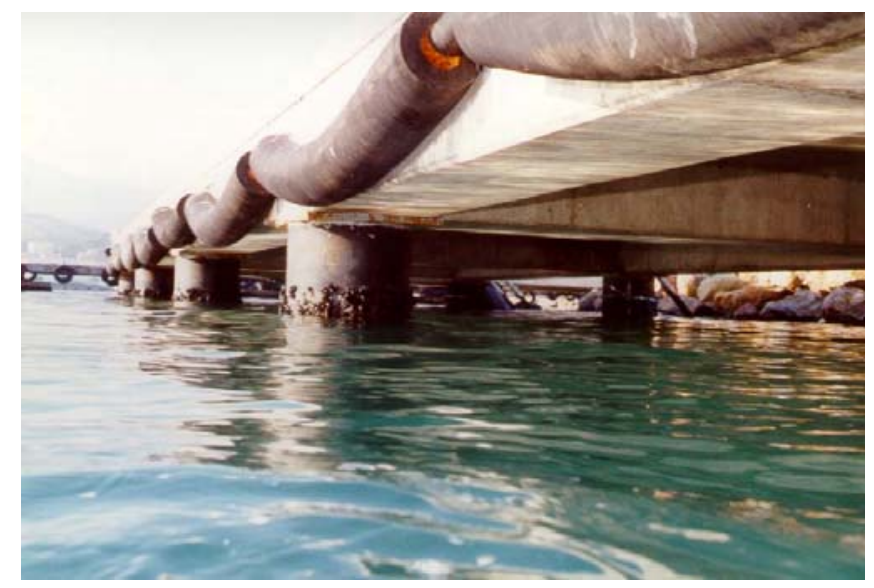

Figure. 5 - Détail du quai 
Retenons que la technique exposée est une solution innovante en terme de prolongation de durée de vie et en terme de diminution de frais et d'interventions ultérieures. En effet, après candidature, instruction est examen du dossier par un groupe d'experts indépendants, le procédé anticorrosion a obtenu le label $\operatorname{IVOR}^{(2)} \mathrm{n}^{\circ} 02.4$ délivré par la DRAST ${ }^{(3)}$.

\section{Références}

1 Barreda H., Guillemard M., (1994). La corrosion des installations portuaires. Actes du colloque d'Aix en $P^{c e}$ - ENSAM sur «La protection et anticorrosion des ouvrages en acier et bétons armé et précontraint en milieux marins» CEFRACOR.

2 Hache A., (1959). La corrosion des métaux. Presses Universitaires de France à Vendôme.

3 Lamoureux J. J., (1994). Précis de corrosion. Editions Beauchemin, Laval province du Québec, Canada.

4 Barreda H., Lazzeri A., (1995). Protection cathodique des palplanches en eau de mer : dimensionnement des anodes sacrificielles. Note technique du bulletin de liaison des Ponts et Chaussées $n^{\circ} 195$ LCPC Paris.

5 Barreda H., Hubert C., Fardella C., (2002). «Systèmes de protection anticorrosion duplex de structures métalliques à la mer». Actes du colloque d'Aix en $\mathrm{P}^{\mathrm{ce}}$ sur la protection cathodique et revêtement associés, CEFRACOR. 